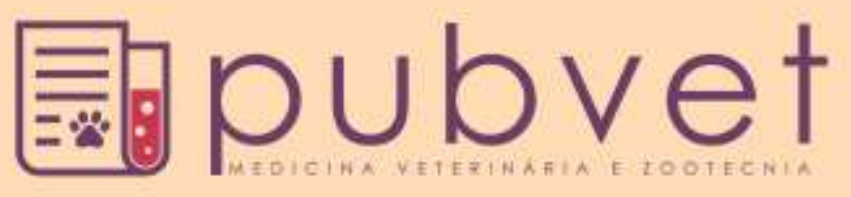

https://doi.org/10.22256/pubvet.v12n1a1.1-7

\title{
Utilização de terapia assistida por animais como ferramenta no tratamento de doenças em humanos: Revisão
}

\author{
Mariana Pereira da Costa ${ }^{\oplus}$, Fábio Gato ${ }^{2}$, Marcio Nogueira Rodrigues ${ }^{\oplus 1}$ \\ ${ }^{I}$ Discente do curso de Medicina Veterinária da Escolar Superior Batista do Amazonas. Manaus-AM Brasil. E-mail: marianafpc94@yahoo.com.br \\ ${ }^{I}$ Professor do curso de Medicina Veterinária da Escolar Superior Batista do Amazonas. Manaus-AM Brasil. E-mail: marcio_medvet@hotmail.com \\ ${ }^{2}$ Médico Veterinário da Pet House \& Cia. Manaus-AM Brasil.E-mail: fabiolopesadestra@gmail.com \\ *Autor para correspondência
}

\begin{abstract}
RESUMO. Este trabalho teve por objetivo atualizar o profissional de Medicina Veterinária quanto a sua função na Terapia Assistida por Animais (TAA), tendo em vista que é um recurso que está sendo cada vez mais utilizado pela medicina humana como parte do processo terapêutico de diversas doenças humanas, seja emocional, física, cognitiva ou social. A TAA tem como ferramenta principal o animal, maioria das ocasiões o cão, portanto o bem-estar desses animais devem ser mantidos, assim como, sua saúde física e mental. O médico veterinário tem uma responsabilidade importante durante esse momento de terapia, logo é essencial manter a integridade física e emocional do cão, assim também como a realização do seu treinamento com o intuito de fornecer um ambiente calmo e rotineiro tanto para os cães quanto para o paciente humano, buscando o sucesso e resposta positiva, pois além de fazer parte do seu campo de atuação, certos conhecimentos e habilidades práticas são cabíveis e exclusivas do profissional médico veterinário.
\end{abstract}

Palavras chave: Bem-estar, cão, zooterapia

\section{Use of animal-assisted therapy as a tool in the treatment of human diseases: Review}

ABSTRACT. The aim of this study was to update the Veterinary Medicine professional regarding its role in animal assisted therapy (TAA), considering that it is a resource that is increasingly being used by human medicine as part of the therapeutic process of several human diseases, like emotional, physical, cognitive or social. The TAA has the animal as the main tool, most occasions is the dog, therefore the welfare of animals need to be kept as well as their physical and mental health. The veterinarian has an important responsibility during this period of therapy therefore it is essential to maintain the physical and emotional integrity of the dog as well as carrying out his training in order to provide a quiet and routine environment for both dogs in order to find the success and positive response during the treatment. It was concluded that the role of the veterinarian during all the elaboration of the activity of extreme validity, besides being part of its field of action, certain knowledge and practical practices are adequate and exclusive of the veterinarian professional.

Key words: Zootherapy, dog, welfare

\section{Uso de la terapia asistida por animales como herramienta en el tratamiento de enfermedades de paciente humano: Revisión}

RESUMEN. Este trabajo tiene por objetivo actualizar el profesional de medicina veterinaria en cuanto a su función en la Terapia asistida por animales (TAA), teniendo en 
vista que es uno recurso que está siendo utilizado cada vez más por la medicina humana como parte del proceso terapéutico de diversas enfermedades humanas, sea emocional, física, cognitiva o social. La TAA tiene como herramienta principal el animal, en la mayoría de los casos el perro, por lo tanto el bienestar de estos animales debe mantenerse, así como su salud física y mental. El veterinario tiene una responsabilidad importante durante este momento de terapia, es esencial mantener la integridad física y emocional del perro, así también como la realización de su entrenamiento con el fin de proporcionar un ambiente tranquilo y rutinario tanto para el perro cuanto para el paciente humano, buscando éxito y respuesta positiva, pues además de formar parte de su campo de actuación, certos conocimientos e habilidades prácticas son cabales y exclusivas del profesional de la medicina veterinaria.

Palabras clave: Bienestar, perro, zooterapia

\section{Introdução}

$\mathrm{O}$ bem-estar animal tem sido bastante pesquisado ao redor do mundo e, como consequência, tem sido produzido conhecimento capaz de fornecer subsídio para a elaboração de diretrizes para os diversos tipos de utilização de animais na terapia assistida. A Terapia Assistida por Animais é um recurso terapêutico em que se utiliza a relação humano-animal com intuito de promover a saúde física, social, emocional e funções cognitivas das pessoas. Essa atividade se baseia no uso de cães terapeutas, os quais devem estar com uma saúde física e mental integra para o contato com os pacientes. O objetivo dessa revisão de literatura é atualizar o médico veterinário em relação a atividade em questão e enfatizar o indispensável papel do profissional durante todo o processo terapêutico que envolve a interação animal-humano ou animal-paciente.

\section{Bem-estar animal}

A associação entre homens e lobos, uma vez que cães são lobos com uma linhagem genética similar, porém domesticados, ocorre há pelo menos 12.000 anos. Ambas as espécies tinham benefícios daquela associação inicial, a qual quase certamente girava em torno de habilidades em relação à caça de presas grandes, e poderia da mesma forma se dizer que cães domesticaram os seres humanos como os seres humanos domesticaram os cães (Broom, 2006). Apesar das frequentes alterações genéticas ocorrerem em todos os cães que se associaram aos seres humanos, vários papéis na sociedade humana foram assumidos pelos cães, e características diferentes foram vantajosas para cada um deles. As raças de cães surgiram inicialmente por causa da demanda por tais papéis (Broom \& Johnson, $\underline{1993}$ ).

Os cães domésticos foram criados seletivamente por vários comportamentos, capacidades, e atributos físicos (Dewey \& Bhagat, 2002). No passado a relação entre humanos e cães era mais prática então humana forneciam comida, abrigo e contato social, enquanto o cão contribuía com o pastoreio, proteção e caça. População de cães domésticos cresceu significativamente com o aumento da urbanização após a segunda guerra mundial. (Derr, 2004).

Com o surgimento dos diferentes tipos de cães, foi empregada uma seleção artificial para fazer que eles tivessem uma aparência um pouco mais impressionante ou extrema em vários aspectos. Raças grandes, pequenas, com pelos longos, focinho curto, patas curtas, corrida extremamente rápida e forte foram desenvolvidas. As pessoas selecionaram cães por características funcionais e não biológicas tanto que foram produzidos cães surdos, quase cegos, incapazes de respirar normalmente, propensos a displasias, entrópio ou dobras de pele excessiva. Todas essas modificações resultaram em baixo grau de bemestar (Broom \& Molento, 2004).

$\mathrm{O}$ bem-estar animal é a condição fisiológica e psicológica na qual o animal de companhia é capaz de adaptar-se comodamente ao entorno, podendo satisfazer suas necessidades básicas e desenvolver suas capacidades conforme a sua natureza biológica (Calderón, 2010).

$\mathrm{O}$ desenvolvimento adicional do bem-estar animal requer produção de conhecimento por parte de especialistas em Comportamento Animal. Para prover boas condições para animais de fazendas, reprodução de espécies ameaçadas de extinção, cuidado apropriado para animais de companhia, é necessária uma forte base de estudo do comportamento.

Bem-estar animal: qual a novidade? A maior novidade é a organização de assuntos segundo uma ordem de prioridade a partir da perspectiva da qualidade de vida dos animais evolvidos, que por 
sua vez permita a busca de estratégias de melhoria do grau de bem-estar animal. Antes da pesquisa e do ensino de bem-estar animal, os assuntos eram trabalhados a partir de um conjunto de valores que raramente envolvia o diagnóstico e a consideração do bem-estar animal for agregada à formação profissional e à atuação nas diversas áreas, inclusive na clínica médica de pequenos animais. As ações em bem-estar animal caracterizam-se pelo objetivo geral de diminuir o sofrimento físico, comportamental e psicológico dos animais sob a nossa guarda (Molento, 2005).

Visando manter o bem-estar do animal, é preciso cuidar, dedicar-se pacientemente. Além de oferecer abrigo, recursos para sobrevivência como água, alimento, proteção, cuidados médicos e terapêuticos. Acolher um animal é um ato respeitoso, e muito mais que isso, é um ato que na medida faz aflorar no ser humano sentimentos como amor incondicional, paciência, responsabilidade, respeito, entre outros, ou seja, tende a formar seres humanos melhores.

A avaliação do bem-estar ou esse excessivo não é fácil e alguns métodos de análise são bastante subjetivos, encontra-se a dosagem de cortisol e mensuração de variações fisiológicas, especialmente pressão arterial. O uso de técnicas não invasivas pode ser considerado a melhor opção para se obter amostras de saliva, urina e fezes, que serão analisadas com o intuito de avaliar o estresse por meio de variações hormonais (Beerda et al., 1996, Queyras \& Carosi, 2003).

\section{Terapia assistida}

Ao longo do tempo, os animais foram aproveitados nas mais variadas funções. Amados, temidos ou usados como fontes de alimento, de proteção contra condições climáticas adversas e, por fim, protegidos dentro de milhões de lares. A história da relação homem-animal percorreu um longo caminho de adestramento, companheirismo e convívio doméstico. A utilização de animais como alternativa de terapia foi iniciada no século XIX, quando médicos clínicos gerais perceberam, entre os pacientes com algum tipo de deficiência mental, benefícios na socialização após o contato com os animais. Em virtude disso, esta terapia começou a ser mais utilizada e passou a ter mais destaque, sendo a técnica, hoje identificada como Terapia Assistida por Animais (TAA) (Mendonça et al., 2014). A Terapia Assistida por Animais é um processo terapêutico formal em âmbito mundial, padronizada pela organização americana
Delta Society. Congrega outras instituições, órgãos certificadores, grupos, cursos e voluntários, sendo que dele participam profissionais da área da saúde humana, animais, seu proprietário ou condutores (Delta Society, 2005).

A terapia assistida por animais (TAA) consiste na utilização de animais como instrumentos facilitadores de abordagem e de estabelecimento de terapias de pacientes, tais como, aqueles com necessidades especiais, crianças com distúrbios cognitivos ou emocionais e idosos. O termo tem sido recomendado pela Delta Society, para conceituar as terapias nas quais o animal é utilizado como motivador, em substituição a outras terminologias menos específicas, como petterapia ou zooterapia (Dotti, 2005; Oliva, 2010). Esse tipo de terapia pode ser inserido nas áreas relacionadas ao desenvolvimento psicomotor, desenvolvimento sensorial, distúrbios físicos, mentais e emocionais, em tratamentos destinados a melhora da socialização, ou ainda na recuperação da autoestima. (Machado et al., 2008). A TAA objetiva a introdução do animal juntamente com o indivíduo ou um grupo onde este fará parte do tratamento, visando sempre promover saúde física, social, emocional e funções cognitivas (Inataa, 2009).

Os recursos da TAA podem ser direcionados a pessoas de diferentes faixas etárias, instituições penais, hospitais, casas de saúde, escolas e clínicas de recuperação. É fundamental o trabalho de uma equipe multidisciplinar capaz de escolher $o$ método mais adequado a ser aplicado, acompanhando as atividades e o bem-estar dos animais e pacientes, que irá refletir no benefício real da qualidade de vida dos mesmos ( $\underline{\operatorname{San}}$ Joaquín, 2002).

Em ambientes hospitalares esta atividade proporciona um relaxamento em relação ao tenso clima que a instituição pode provocar ao paciente, trazendo uma melhora na interação entre paciente e a equipe de saúde. Diante disso, analogicamente, esta prática também pode ser benéfica em se tratando de atendimento odontopediátrico, pois possibilita a mesma sensação de bem-estar e, diante disso, uma melhora na comunicação entre os agentes envolvidos (Kobayashi et al., 2009). Reconhecida em diversos países do mundo, essa terapia é comprovadamente uma técnica útil na socialização de pessoas, na psicoterapia, em tratamentos de pacientes com necessidades especiais e na diminuição da ansiedade de várias 
causas. Essa abordagem terapêutica em pacientes idosos e com necessidades especiais também oferece auxílio terapêutico a pacientes com doenças graves. Tal atividade baseia-se no uso de animais chamados co-terapeutas, os quais devem apresentar boa saúde física e mental visando ao bem-estar do animal e à segurança do paciente assistido quanto a riscos de zoonoses e comportamentos inadequados (Oliva, 2007).

Estudos desenvolvidos mostram que o simples contato com o animal já é suficiente para promover bem-estar. Alguns benefícios da TAA foram comprovados, como a diminuição da ansiedade e a melhora do humor (Costa, 2000). O relacionamento com animais, em especial com os cães, promete uma série de benefícios, alguns deles já bem pesquisados; outros ainda aguardam confirmações científicas. Os benefícios verificados são vários: proporciona o aumento de exercícios físicos e, consequentemente, a melhora do estado geral da saúde cardiovascular; promove interação com outras pessoas; apresenta um efeito antiestresse; representa um suporte social e de afeto, entre outros (Chelini \& Otta, 2016).

No estudo de Redefer \& Goodman (1989), observou-se que a fuga ao contato e isolamento diminuía significativamente na presença do cão de terapia, enquanto que as interações iniciadas pela criança com o terapeuta aumentavam em comparação com as sessões em que o cão não estava presente. A criança aliava-se ao terapeuta em jogos simples, verificando-se aumento do contato físico, afetivo e imitação das ações do terapeuta. As crianças mostravam menos comportamentos negativos e repetitivos (postura das mãos, zumbidos e outros ruídos produzidos) demonstrando outro comportamento socialmente mais apropriado (Redefer \& Goodman, 1989).

Os aspectos sociais, físicos e emocionais estão praticamente conectados e promovem também resultados mentais positivos, estimulando memória antiga e recente em idosos. Cada ser humano tem seu perfil e limites quanto à saúde, sentimentos e personalidade (Dotti, 2005). Além dos efeitos psicológicos, os animais também podem trazer benefícios fisiológicos para as pessoas. Alguns dos benefícios descritos são a redução dos níveis de triglicérides, colesterol, pressão sanguínea e estresse, diminuição da incidência de doenças cardiovasculares e maior facilidade de recuperação em caso de doenças (Jofré, 2005).

\section{A utilização de animais em processos de terapia assistida e a importância do acompanhamento por veterinário}

Em anos recentes, a capacidade dos animais para irem além do papel de bicho de estimação e atuarem como curador dos seus guardiões humanos foi demonstrado na pesquisa, ainda que com literatura limitada. Apesar de as atividades (AAA) e a terapia (AAT) assistida por animais não serem tipos comuns de tratamentos complementares usados em pessoas com condições crônicas foram investigadas e consideradas valiosas em vários contextos, tais como hospitalares, terapêuticos, educacionais e de moradia assistida, particularmente entre crianças e idosos (Caprilli \& Messeri, 2006, Kanamori et al., 2001, Trotter et al., 2008).

Telhado (2001) relata que podem ser utilizados todos os tipos de animais que possam entrar em contato com os humanos sem oferecer-lhes perigo sendo os mais utilizados o gato, coelho, tartaruga, chinchila, hamster, peixe, furão, pássaro e até mesmo animais exóticos como a iguana. Para que os animais façam parte da terapia, é realizado um conjunto de exames rigorosos para garantir que a saúde do animal esteja 100\%. Devem apresentar bom comportamento, serem sociáveis com pessoas estranhas e habituadas com o convívio de outros animais. No caso dos cães, podem participar os de pequeno e grande porte (que deve obedecer a comandos básicos de obediência) e de qualquer raça. Animais muito jovens possuem dentes e unhas afiadas, os muitos velhos se cansam ao término da visita, por isso não devem participar da terapia.

O cão é o principal animal, pois apresenta uma natural afeição pelas pessoas, é adestrado facilmente, cria respostas positivas ao toque e possui uma grande aceitação por parte das pessoas. Estudos demonstram que animais que podem ser tocados resultam numa terapia mais efetiva. Relatos referentes a algumas patologias mostram que esta interação cão-paciente melhora o padrão cardiovascular, diminuindo a pressão arterial e os níveis de colesterol. Tal interação também produz o aumento na concentração plasmática de endorfinas, ocitocinas, prolactina, dopamina e diminui concentração plasmática de cortisol, substâncias que atuam positivamente no estado de ansiedade (Jofré, 2005).

Para que o cão possa participar desta terapia, deve haver uma intensa e constante preocupação com a seleção e saúde desse animal, sendo 
necessária a avaliação por três profissionais, a saber: um veterinário, um psicólogo com especialização em comportamento canino e um adestrador. O primeiro é responsável pela verificação da saúde física do animal; o segundo, pela avaliação do comportamento quanto à socialização, obediência e temperamento do animal; o terceiro vem com a parte do adestramento dos cães, ensinando aos animais como se comportar e usar técnicas e habilidade para lidar com os pacientes que irão participar (Dotti, 2005).

É essencial que o animal coterapeuta seja acompanhado em todas as sessões por alguém que se responsabilize exclusivamente por ele, não só por sua atuação nas atividades, mas também pela manutenção de sua segurança e bem-estar durante todo o tempo. Tal condutor deve receber treinamento adequado em comportamento, além de conhecer profundamente a espécie e o indivíduo com quem está trabalhando. É importante que o condutor saiba quais comportamentos podem ser considerados normais para aquele indivíduo e esteja atento para mudanças comportamentais e para exibição de comportamentos que indiquem desconforto (Chelini \& Otta, 2016).

Sendo um programa multidisciplinar, sabe também a participação do médico veterinário, sua participação é indispensável para o desenvolvimento do programa de zooterapia. Sendo assim, este profissional desempenha papel essencial no sentido de acompanhar as manifestações comportamentais do animal junto a adestradores e etólogos, assim como, no sentido de zelar pela saúde animal, manter os animais em perfeito estado de saúde, com objetivo de melhorar sua qualidade de vida. Além de cuidados básicos, como alimentação balanceada, cuidados necessários para o bem-estar do animal, higiene, vacinações e vermifugações periódicas, mecanismos de controle de ectoparasitas (Santos, 2006, Anderline, 2007).

Nesse caso o veterinário desempenha um papel fundamental no sentido de acompanhar as manifestações comportamentais do animal, assim como também zelar pela sua saúde. A Organização Mundial reconhece a importância dos animais na zooterapeutas. Por isso não pode deixar de se preocupar com a saúde física do animal, que é um aspecto indispensável que visa não somente o bom desempenho e bem-estar animal, mas também a garantia de que não haverá riscos de transmissão de zoonoses e contaminação do local onde a terapia será realizada (Anderline, 2007).

Dentre as sugestões de manejo sanitário estão a vacinação anual com vacina polivalente, vacinação anual contra Raiva, vacinação contra Traqueobronquite Canina, vermifugação a cada quatro meses com vermicidas de amplo espectro, exame parasitológico semestral (sempre com resultado negativo), banho no mínimo $48 \mathrm{~h}$ antes das visitas, controle de ectoparasitas, limpeza de dentes e ouvidos, sem sinais de enfermidades (Flôres, 2009).

Para realizar os testes devem ser seguidos alguns critérios para avaliar o animal, como chamar (atração por pessoas), acompanhar (seguir a liderança humana), restrição (facilidade de controle sob domínio físico), acariciar (facilidade de controle pelo carinho), elevação (facilidade de controle em situação de risco), buscar (vontade de fazer algo pelo dono), pressão na pata (resistência a dor), barulhos forte (resistência a sons), perseguir (reação a algo que se move), pegar de surpresa (reação a situação inesperada). Depois da avaliação e seleção do animal, deve realizar uma combinação com a classe de pessoas que os mesmos irão interagir (Flôres, 2009).

\section{Considerações Finais}

A terapia assistida por animais tem tido seus benefícios e eficácia comprovada através dos diversos estudos e seus autores. Assim como a necessidade do médico veterinário estar envolvido durante todo o processo para que o bem-estar do cão seja sempre mantido e haja melhora significativa dos pacientes quanto às suas respectivas doenças.

\section{Referências Bibliográficas}

Anderline, G. A. 2007. Benefícios do envolvimento do animal de companhia (cão e gato), na terapia, na socialização e bem estar das pessoas e o papel do médico veterinário. Revista do Conselho Federal de Medicina Veterinária, 13, 70-75.

Beerda, B., Schilder, M. B. H., Janssen, N. S. C. R. M. \& Mol, J. A. 1996. The use of saliva cortisol, urinary cortisol, and catecholamine measurements for a noninvasive assessment of stress responses in dogs. Hormones and behavior, 30, 272-279. 
Broom, D. M. 2006. Behaviour and welfare in relation to pathology. Applied Animal Behaviour Science, 97, 73-83.

Broom, D. M. \& Johnson, K. G. 1993. Stress and animal welfare. Springer Science \& Business Media, London.

Broom, D. M. \& Molento, C. F. M. 2004. Animal welfare: concept and related issues-review. Archives of Veterinary Science, 9, 1-11.

Calderón, N. 2010. Bienestar Animal. Revista de la Academia Colombiana de Ciencias Veterinarias, 1, 50.

Caprilli, S. \& Messeri, A. 2006. Animal-assisted activity at A. Meyer Children's Hospital: a pilot study. Evidence-Based Complementary and Alternative Medicine, 3, 379-383.

Chelini, M. O. M \& Otta, E. Terapia assistida por animais - Barueri, SP: Manole, 2016.

Costa, R. M. E. M. Ambientes virtuais na reabilitação cognitiva de pacientes neurológicos e psiquiátricos. Tese (D. Sc., Coppe Sistemas) - UFRJ, Rio de Janeiro, 30p., 2000.

DELTA SOCIETY. Atividade e terapia por animais. A/TAA [texto na internet] 2005 [citado 2005 Mar 19]. Disponível em: http://www.projetocao.com.br/main.htm.

Derr, M. Dog's best friend. Vol.2. Chicago: University of Chicago Press, 2004.

Dewey, T. and Bhagat, S. Canis lupus familiaris(Online). Animal Diversity Web, 2002.

Dotti, J. Terapia e animais. São Paulo: Noética, 2005.

Flôres, N. L, Os benefícios da interação homemanimal e o papel do médico veterinário, Porto Alegre, RS, 2009.

Instituto Nacional de Ações e Terapias Assistidas por Cães; TAA - Terapia Assistida por Animais [texto na internet] 2011 [citado 2011 Jun 2011]. Disponível em: http:www.inataa.org.br

Jofré, M. 2005. Visita terapéutica de mascotas en hospitales. Revista Chilena de Infectología, 22, 257-263.

Kanamori, M., Suzuki, M., Yamamoto, K., Kanda, M., Matsui, Y., Kojima, E., Fukawa, H., Sugita, T. \& Oshiro, H. 2001. A day care program and evaluation of animal-assisted therapy (AAT) for the elderly with senile dementia. American Journal of Alzheimer's Disease \& Other Dementias, 16, 234-239.

Kobayashi, C. T., Ushiyama, S. T., Fakih, F. T., Robles, R. A., Carneiro, I. A. \& Carmagnani, M. I. S. 2009. Development and implementation of Animals-Assisted Therapy in a universitary hospital. Revista brasileira de enfermagem, 62, 632-636.

Machado, J. D. A. C., Rocha, J. R., Santos, L. M. \& Piccinin, A. 2008. Terapia assistida por animais (TAA). Revista Científica Eletrônica de Medicina Veterinária, 10, 1-7.

Mendonça, M. E. F., Silva, R. R., Sá, F. M. J. \& Peixoto, S. P. L. 2014. A terapia assistida por cães no desenvolvimento socioafetivo de crianças com deficiência intelectual. Caderno de Graduação-Ciências Biológicas e da Saúde-UNIT-ALAGOAS, 2, 11-30.

Molento, C. F. M. 2005. Bem-estar e produção animal: Aspectos econômicos - Revisão. Archives of Veterinary Science, 10, 1-11.

Oliva, V. N. L. S.; Júnior, A. B .S.; Carvalho, E .A. G. Experiências clínicas do projeto cãocidadão-unesp no hospital neurológico Ritinha Prates - Araçatuba - SP, 2007.

Oliva, V. N. L. S. Terapia Assistida por Animais. In: Costa Neto, E. M. \& Alves, R. R. N. Zooterapia - os animais na medicina popular brasileira, 1.ed. Recife: NUPEEA, 2010. p.127-140

Queyras, A. \& Carosi, M. 2003. Non-invasive techniques for analysing hormonal indicators of stress. Annali Dell'Istituto Superiore di Sanita, 40, 211-221.

Redefer, A. \& Goodman, A. J. F. 1989. Pet facilitated therapy with autistic children. Journal of Autism and Developmental Disorders, 19:461-467.

Reed. R; Ferrer. L \& Villegas. N, 2012. Curadores Naturais: uma revisão da terapia e atividades assistidas por animais como tratamento complementar de doenças crônicas. Revista Latino-AM de Enfermagem.

San Joaquín, M. P. Z. 2002. Terapia asistida por animales de conpañia. Bienestar para el ser humano. Temas de Hoy, 1, 143-149.

Santos, K. Terapia Assistida por animais; uma experiência além da ciência/Karen Cristini dos Santos. São Paulo: Editora Paulinas, 2006 coleção Vida assistida. 
Telhado, J. 2001. Animais ajudam a curar doenças. Jornal do Brasil, 9, 1.

Trotter, K. S., Chandler, C. K., Goodwin-Bond, D. \& Casey, J. 2008. A comparative study of the efficacy of group equine assisted counseling with at-risk children and adolescents. Journal of Creativity in Mental Health, 3, 254-284.
Article History:

Received 6 June 2017

Accepted 22 July 2017

Available on line 21 October 2017

License information: This is an open-access article distributed under the terms of the Creative Commons Attribution License 4.0, which permits unrestricted use, distribution, and reproduction in any medium, provided the original work is properly cited. 